\title{
Carbapenemasas en aislamientos de Pseudomonas aeruginosa resistentes a carbapenémicos aisladas en hospitales de Chile
}

\author{
Carbapenemases produced by Carbapenem-resistant Pseudomonas aeruginosa \\ isolated from hospitals in Chile
}

\begin{abstract}
Jandira S. Tomás da Costa', Celia A. Lima ${ }^{1,3,4}$, Alejandra Vera-Leiva ${ }^{1}$, Iván San Martin Magdalena', Helia Bello-Toledo 1,4, Mariana Domínguez Yévenes ${ }^{1}$, Andrés Opazo-Capurro ${ }^{1,4}$, Sergio Mella Montecinos 2,5,6, Mario Quezada-Aguiluz',2,4, Gerardo González-Rocha1,4 y Grupo Colaborativo de Resistencia\#
\end{abstract}

\author{
${ }^{1}$ Laboratorio de Investigación en Agentes Antibacterianos (LIAA). Departamento de Microbiología, Facultad de Ciencias Biológicas, Universidad de Concepción. \\ ${ }^{2}$ Departamento de Medicina Interna. Facultad de Medicina, Universidad de Concepción. \\ ${ }^{3}$ Departamento Prevención y Salud Pública Odontológica, Facultad de Odontología, Universidad de Concepción. \\ ${ }^{4}$ Millennium Nucleus on Interdisciplinary Approach to Antimicrobial Resistance (MICROB-R), Chile. \\ ${ }^{5}$ Unidad de Medicina Interna, Hospital Traumatológico de Concepción. \\ "Unidad de Infectología, Hospital Regional "Dr. Guillermo Grant B.", Concepción.
}

No existen conflictos de interés.

Esta investigación ha sido financiada por Programa Atracción e Inserción de Capital Humano Avanzado (PAI), Proyecto N PAI79170082 y Beca AGCID- Nelson Mandela a JST.

Recibido: 6 de febrero de 2020 (segunda versión: 11 de diciembre de 2020) / Aceptado: 11 de diciembre de 2020

\section{Resumen}

Introducción: La resistencia a carbapenémicos mediada por carbapenemasas en Pseudomonas aeruginosa es un mecanismo importante; sin embargo, la pérdida de la porina OprD continúa siendo el mecanismo más frecuente. Objetivo: Determinar la proporción de aislados de $P$. aeruginosa, resistentes a imipenem y/o meropenem, productores de carbapenemasas, el tipo de enzima producida y la relación genética entre los aislados. Material y Métodos: Se incluyó 113 aislados resistentes al menos a un carbapenémico, provenientes de 12 hospitales de 9 ciudades de Chile. Adicionalmente se determinó la susceptibilidad a ceftazidima, amikacina, gentamicina, piperacilina/ tazobactam, ciprofloxacina y colistina. Se realizó Carba NP y en los aislados positivos (n: 61) se detectó genes de carbapenemasas por RPC. Los aislados fueron tipificados por restricción con SpeI y PFGE. Resultados: No todos los aislados presentan carbapenemasas, y sólo en $61 / 113$ de ellos (54\%) se amplificó bla $a_{\mathrm{KPC}}$ (32) o bla ${ }_{\mathrm{VIM}}$ (29). En

\begin{abstract}
Background: Carbapenem resistance mediated by carbapenemases in Pseudomonas aeruginosa is an important mechanism; however, loss of porin OprD remains as the most frequent. Aim: To determine the proportion of $P$. aeruginosa isolates, resistant to imipenem and/or meropenem, producing carbapenemases, the type of enzyme produced and the genetic relationship between the isolates. Methods: One hundred and thirteen resistant to at least one carbapenem isolates, obtained in 12 hospitals and 9 cities in Chile were studied. Additionally, susceptibility to ceftazidime, amikacin, gentamicin, piperacillin/tazobactam, ciprofloxacin and colistin was determined. Carba NP was performed and in the positive isolates carbapenemase genes were detected by PCR. The isolates were typified by restriction with SpeI and PFGE. Results: Not all isolates produce carbapenemases, and only in 61/113 of them $(54 \%)$ the $b l a_{\mathrm{KPC}}(32)$ or $b l a_{\mathrm{VIM}}(29)$ was amplified. In none of the isolates was found the coharboring of both genes. The pulso-
\end{abstract}

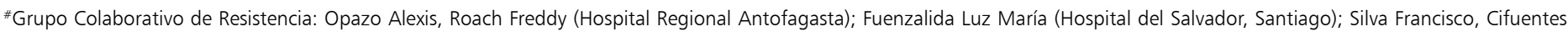

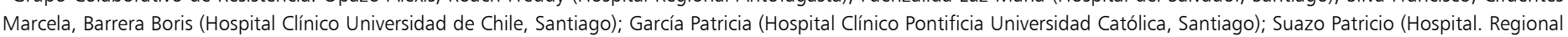

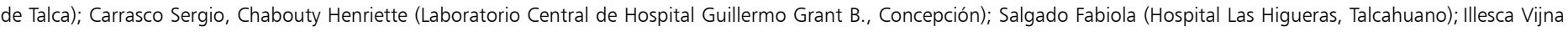
(Hospital Hernán Henríquez, Temuco); Rioseco María Luisa, Mella Andrea (Hospital Base Puerto Montt).

\author{
Correspondencia a: \\ Gerardo González-Rocha \\ ggonzal@udec.cl
}


ninguno de los aislados se encontró co-portación de ambos genes. Los pulsotipos indican que no hay diseminación clonal de los aislados, evidenciando una importante diversidad genética. Conclusiones: Los aislados de $P$. aeruginosa productores de carbapenemasas, obtenidos en hospitales de Chile, portan genes $b l a_{\mathrm{KPC}}$ y $b l a_{\mathrm{VIM}} \mathrm{y}$, en su mayoría, son policlonales. Estos resultados ponen énfasis en la importancia de realizar estudios epidemiológicos con mayor número de aislados que permitan conocer mejor la epidemiología de $P$. aeruginosa productoras de carbapenemasas en Chile.

Palabras clave: carbapenémicos; carbapenemasas; Pseudomonas aeruginosa; tipificación molecular; Chile.

\section{Introducción}

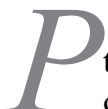
seudomonas aeruginosa es un bacilo gramnegativo no fermentador de glucosa, aerobio estricto, reconocido como patógeno oportunista, que afecta principalmente a pacientes de Unidades de Cuidados Intensivos (UCIs), pacientes inmunodeprimidos y pacientes con fibrosis quística, ocasionando infecciones asociadas a la atención en salud (IAAS) y neumonía asociada a ventilación mecánica $(\mathrm{NAVM})^{1,2}$.

Recientemente, $P$. aeruginosa resistente a carbapenémicos se ha incorporado a la lista de patógenos prioritarios de la Organización Mundial de la Salud (OMS), para investigación y desarrollo de nuevos antimicrobianos y ha sido catalogada con prioridad crítica ${ }^{3}$. De acuerdo al Ministerio de Salud (MINSAL) ${ }^{4}$, en Chile esta bacteria se ubica en el primer lugar como agente etiológico de NAVM en adultos y, en segundo lugar, en casos pediátricos ${ }^{3}$. Además, se informa su importante aislamiento de otros cuadros clínicos como infección urinaria en pacientes con catéter urinario permanente (CUP), en herida operatoria e infecciones del torrente sanguíneo, especialmente en pacientes inmunodeprimidos donde corresponde al segundo microorganismo más frecuentemente aislado ${ }^{4}$. Actualmente, el aislamiento de cepas de $P$. aeruginosa resistentes a carbapenémicos, grupo de antimicrobianos que representan la última opción terapéutica, ha sido reconocido por la OMS como un serio problema a nivel global, que conlleva a infecciones con alta tasa de mortalidad, prolongadas estadías hospitalarias y elevados costos en el sistema de salud ${ }^{3}$.

La resistencia a carbapenémicos en $P$. aeruginosa puede estar mediada por múltiples mecanismos ${ }^{2}$. Sin embargo, la capacidad de sintetizar enzimas capaces de hidrolizar estos compuestos, denominadas carbapenemasas, constituye actualmente el mecanismo de resistencia más importante, debido a la capacidad de diseminación horizontal que pueden presentar los genes codificantes de estas enzimas.

En Chile, existe muy poca información acerca de la resistencia enzimática a imipenem y meropenem exhibida por cepas de $P$. aeruginosa $a^{5-7}$, y por esto, el objetivo de este estudio fue determinar el tipo de carbapenemasa en un grupo de aislados de $P$. aeruginosa resistentes a carbapenémicos y la relación genética entre los aislados productores de carbapenemasas. types indicated no clonal dissemination of the isolates, evidencing an important genetic diversity. Conclusions: $P$. aeruginosa isolates producing carbapenemases, obtained in Chilean hospitals carry $b l a_{\mathrm{KPC}}$ and $b l a_{\mathrm{vIM}}$ genes and, mostly, are polyclonal. These results emphasize the importance of carrying out epidemiological studies with a greater number of isolates to allow a better understanding of the epidemiology of carbapenemase-producing $P$. aeruginosa in Chile.

Keywords: carbapenems; carbapenemases; Pseudomonas aeruginosa; molecular typing; Chile.

\section{Metodología}

\section{Aislados bacterianos}

Se incluyó 113 aislados de $P$. aeruginosa resistentes a imipenem y/o meropenem, obtenidos entre los años 1998 y 2019 en 12 hospitales de 9 ciudades de Chile: Antofagasta 21 (18,6\%), Santiago 34 (30,1 $\%)$, Concepción 11 (9,7\%), Talcahuano 7 (6,2\%), Temuco $23(20,4$ $\%)$, Puerto Montt $8(7,1 \%)$ y 9 aislados ( $8 \%)$ provenientes de otras ciudades, que incluyen Talca, Lota y Los Ángeles.

Los aislados de $P$. aeruginosa fueron recuperados principalmente de heridas/abscesos $(17 \%)$, tracto respiratorio (16\%), catéter/sonda $(11,8 \%)$, sangre/orina (11\%), hisopado rectal $(4,2 \%)$ y en $39,8 \%$ de los aislados no se tuvo acceso al origen de la muestra.

\section{Susceptibilidad in vitro a los antimicrobianos}

El comportamiento de los aislados a los antimicrobianos fue evaluado mediante la determinación, por dilución seriada en agar, de la concentración inhibitoria mínima (CIM) de imipenem (IPM), meropenem (MEM), amikacina (AMK), gentamicina (GEN), ciprofloxacina (CIP), cefotaxima (CTX) y piperacilina/tazobactam (PIP/TAZO), siguiendo las recomendaciones y puntos de corte propuestos por el Clinical Laboratory Standards Institute (CLSI 2015, CLSI 2019) $)^{8,9}$. Se empleó como control la cepa $P$. aeruginosa ATCC 27853. El comportamiento frente a colistina se determinó mediante el método Colistín Agar-Spot test (CAST) propuesto por el Servicio de Antimicrobianos, INEI ANLIS “Dr. Carlos G. Malbrán” de Argentina (2017) ${ }^{10}$.

\section{Producción y genes de carbapenemasas}

En los 113 aislados se investigó la producción de carbapenemasas por el método de Carba NP descrito por Dortet y cols. (2014) ${ }^{11}$. En aquellos aislados que presentaron una prueba fenotípica positiva se investigaron mediante reacción de polimerasa en cadena (RPC) los siguientes genes que codifican carbapenemasas: $b l a_{\mathrm{VIM}}, b l a_{\mathrm{GES}}, b l a_{\mathrm{KPC}}$, $b l a_{\mathrm{IMP}}, b l a_{\mathrm{GIM}}, b l a_{\mathrm{SPM}}, b l a_{\mathrm{NDM}}$ y $b l a_{\mathrm{OXA}-48}$, de acuerdo al protocolo descrito por Poirel y cols. ${ }^{12}$. Los fragmentos de ADN amplificados se separaron en un gel de agarosa al $2 \%$ conteniendo $1 x$ SafeView ${ }^{\text {TM }}$ Plus (Applied Biological Material, Inc.) a $100 \mathrm{~V}$ durante 45 min en 0,5x de búfer TAE. Los productos de amplificación se enviaron a secuenciar a la compañía MacroGen Corp. U.S.A (9700 Great Seneca Highway, 
Rockville, MD 20850). Posteriormente, se realizaron análisis bio-informáticos comparando las secuencias obtenidas con aquellas informadas en la base de datos del National Center for Biotechnology Information (NCBI), utilizando la herramienta BLAST y el programa MEGA 5 y MEGA 6.

\section{Tipificación molecular de los aislados productores de carbapenemasa}

Se realizó tipificación molecular mediante macrorestricción del genoma bacteriano con la enzima de corte infrecuente SpeI (30 units/ $\mu \mathrm{L}$; Promega Corp., Madison, USA) y posterior separación de los fragmentos producidos por electroforesis de campo pulsado (PFGE, del inglés pulsed-field gel electrophoresis) de acuerdo al protocolo de Jordan y Dalmasso (2015) ${ }^{13}$. La cepa Salmonella serovar Braenderup H9812 (ATCC BAA 664) se digirió con XbaI (10 units/ $\mu \mathrm{L}$; Thermo Scientific, Massachusetts, E.U.A.) y se usó como estándar de tamaño. El análisis de los geles se realizó con el programa BioNumerics v. 6.611 (AppliedMaths, Inc., Austin, TX, E.U.A.) comparando los patrones de corte mediante el coeficiente de Dice y construyendo el dendograma de las cepas por UPGMA (del inglés unweighted pair-group method with arithmetic means). Los parámetros de análisis utilizados fueron: tolerancia $1,5 \%$ y optimización $1 \%$. Las cepas se clasificaron como "genéticamente relacionadas" cuando presentaron un valor mayor o igual a $80 \%$ de relación entre ellas; y como "clonales" cuando presentaron $100 \%$ de identidad.

\section{Resultados}

En 61 de los 113 aislados, el test Carba NP fue positivo, indicando la producción de alguna carbapenemasa y en 52 de ellos no se detectó la producción de estas $\beta$-lactamasas. Los aislados se separaron en tres grupos: productores de VIM (n: 29), productores de KPC (n: 32) y no productores de carbapenemasas (n: 52). El gen $b l a_{\mathrm{KPC}}$ fue amplificado en 32 aislados $(52,5 \%)$ y el gen $b l a_{\mathrm{VIM}}$ en $29(47,5 \%)$, y en ninguno de ellos se detectó la co-portación de ambos genes.

La resistencia a los antimicrobianos ensayados se muestra en la Tabla 1. Se puede observar que el porcentaje de aislados resistentes a IPM es mayor en aquellos productores de alguna carbapenemasa, con un valor de CIM mayor en los productores de KPC (2-1.024 $\mu \mathrm{g}$ / $\mathrm{mL})$. En cambio, el porcentaje de aislados resistentes a MEM es elevado en los tres grupos (> 86\%) y nuevamente la CIM a este carbapenémico es más elevada en los aislados KPC (+) (8-1.024 $\mu \mathrm{g} / \mathrm{mL})$. Respecto de los otros antimicrobianos ensayados, la resistencia a CIP es elevada y similar, tanto en los aislados productores como no productores de carbapenemasas ( $>90 \%$ ), con valores de $\mathrm{CIM}_{90}$ que variaron entre 32 y $128 \mu \mathrm{g} / \mathrm{mL}$. Para AMK se presenta el menor porcentaje de aislados resistentes (20,8-36,7\%) en los tres grupos estudiados y para GEN sólo se observó un elevado porcentaje de resistencia $(75 \%)$ en los aislados VIM (+). Se observó un elevado porcentaje de aislados resistentes a TPZ en el grupo KPC $(+)(76,7 \%)$; en cambio, en los aislados VIM (+) la resistencia alcanzó sólo a 14,3\%; sin embargo, en este grupo el porcentaje de aislados con susceptibilidad intermedia alcanzó a 64,3\%. La resistencia a CAZ fue mayor entre los aislados productores de carbapenemasas, con $64,3 \%$ para aislados VIM (+) y 83,3\% para aislados $\mathrm{KPC}(+)$. En los aislados no productores de carbapenemasas, la resistencia a esta cefalosporina sólo alcanzó Tabla 1. Actividad de agentes antibacterianos sobre aislados hospitalarios de
Pseudomonas aeruginosa resistentes a carbapenémicos

\begin{tabular}{|c|c|c|c|c|}
\hline \multirow[b]{2}{*}{ Antibacterianos } & \multicolumn{4}{|c|}{ Cepas VIM (+) } \\
\hline & Rango CIM & $\mathrm{CIM}_{50}$ & $\mathrm{CIM}_{90}$ & \% cepas $\mathbf{R}$ \\
\hline GEN & $\leq 1->1.024$ & $>128$ & 1.024 & 75,0 \\
\hline AMK & $4->256$ & 32 & $>256$ & 35,7 \\
\hline CIP & $2->128$ & 32 & 128 & 100,0 \\
\hline CAZ & $\leq 8-128$ & 32 & 64 & 64,3 \\
\hline TPZ & $8-256$ & 32 & 128 & 14,3 \\
\hline IPM & $2-256$ & 16 & 256 & 89,7 \\
\hline \multirow[t]{2}{*}{ MEM } & $4-128$ & 64 & 128 & 96,6 \\
\hline & \multicolumn{4}{|c|}{ Cepas KPC (+) } \\
\hline Antibacterianos & Rango CIM & $\mathrm{CIM}_{50}$ & $\mathrm{CIM}_{90}$ & \% cepas $\mathbf{R}$ \\
\hline GEN & $2->128$ & 8 & 64 & 23,3 \\
\hline AMK & $\leq 2>256$ & $\leq 16$ & 128 & 36,7 \\
\hline CIP & $\leq 4->128$ & 32 & 64 & 93,3 \\
\hline CAZ & $8-256$ & 32 & 64 & 83,3 \\
\hline TPZ & $\leq 8->256$ & 256 & $>256$ & 76,7 \\
\hline IPM & $2-1.024$ & 256 & 256 & 93,8 \\
\hline \multirow[t]{2}{*}{ MEM } & $8-1.024$ & 256 & 256 & 100,0 \\
\hline & \multicolumn{4}{|c|}{ Cepas sin carbapenemasa } \\
\hline Antibacterianos & Rango CIM & $\mathrm{CIM}_{50}$ & $\mathrm{CIM}_{90}$ & \% cepas $\mathbf{R}$ \\
\hline GEN & $2->1.024$ & $\leq 8$ & $>128$ & 27,1 \\
\hline AMK & $\leq 2->256$ & $\leq 16$ & 128 & 20,8 \\
\hline CIP & $2->128$ & 4 & 32 & 100,0 \\
\hline CAZ & $\leq 2->1.024$ & 16 & 64 & 37,5 \\
\hline TPZ & $\leq 8-256$ & 32 & 128 & 27,1 \\
\hline IPM & $2-256$ & 8 & 64 & 53,8 \\
\hline MEM & $2-256$ & 16 & 128 & 86,5 \\
\hline
\end{tabular}


a $37,5 \%$. Al ensayar el colistin agar-spot test, todos los aislados fueron no resistentes a colistina ( $\mathrm{CIM} \leq 3 \mu \mathrm{g} /$ $\mathrm{mL}$ ). El análisis molecular de los genes de carbapenemasas indica que la variante producida por los aislados productores de KPC corresponde al alelo $b l a_{\mathrm{KPC}-2} \mathrm{y}$ para los aislados productores de VIM a $b l a_{\mathrm{VIM}-2}$.

La tipificación molecular (PFGE) de 36 aislados de $P$. aeruginosa productores de carbapenemasas (Figura 1) da cuenta que mayoritariamente existe policlonalidad en este grupo de aislados obtenidos en los hospitales de Chile.
Se observan 7 clústeres que agrupan a 21 cepas, aisladas en hospitales de Santiago, Temuco y Puerto Montt, y en sólo uno de ellos (clúster VI) hay dos cepas productoras de KPC que corresponde a un clon aislado en la ciudad de Temuco (P372 y P383), que se relaciona en $83 \%$ con un aislado productor de la misma carbapenemasa, proveniente de la ciudad de Santiago (P345). Los clústeres IV y $\mathrm{V}$, a su vez, se subdividen en sub-clústeres con identidad mayor a $82 \%$ y compuestos por aislados productores de KPC, provenientes principalmente de Temuco.
Figura 1. Dendrograma de aislados de Pseudomonas aeruginosa productores de carbapenemasas provenientes de hospitales de Chile, usando Spel y PFGE. HRA: Antofagasta; HCUCH, HES, HCPUC: Santiago; HRDT: Talca; HDT: Temuco; HPM: Puerto Montt. Se usó $80 \%$ de similitud para definir cepas genéticamente relacionadas. I-VII: clústeres de cepas relacionadas genéticamente.

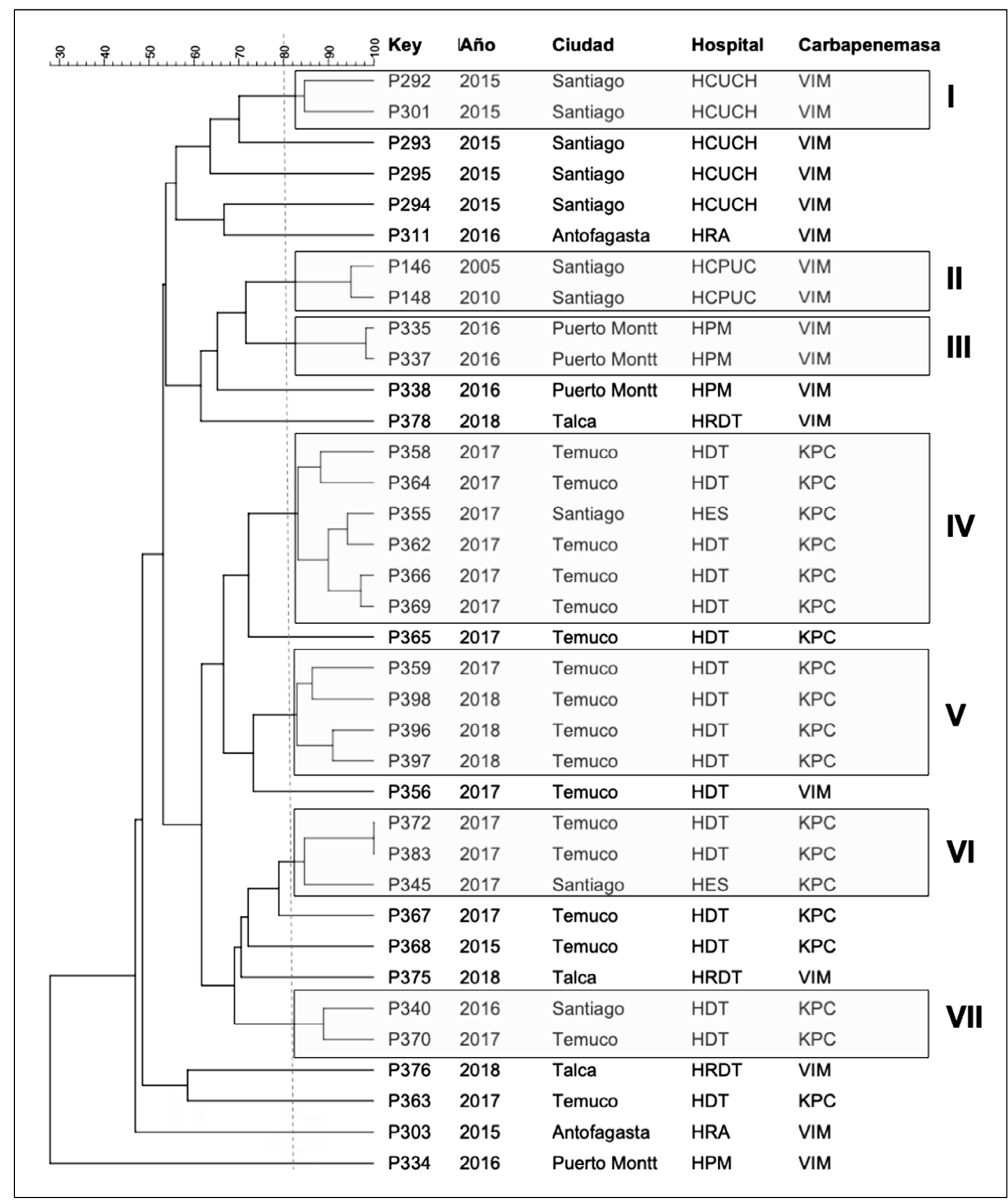


aeruginosa aisladas en Chile en-23 $^{21}$ Ninguno de los otros genes investigados amplificó en los aislados del estudio, dando cuenta que la diversidad de carbapenemasas en $P$. aeruginosa es aún limitada en Chile, una situación que difiere de lo informado para Latinoamérica ${ }^{6}$. El estudio molecular de las variantes alélicas de las carbapenemasas permitió afirmar que éstas corresponden a VIM-2 y KPC-2, concordando con lo que mayoritariamente se ha descrito para Latinoamérica y el mundo ${ }^{6,14}$. Es importante destacar que el porcentaje de aislados no productores de alguna carbapenemasa no es despreciable (46\%), lo que es especialmente importante porque las infecciones causadas por este grupo de aislados podrían ser tratadas con combinaciones como ceftolozano/tazobactam, ya que se ha demostrado que ceftolozano (cefalosporina antipseudomonas) no se ve afectada por mecanismos como la pérdida de la porina OprD y la sobre-expresión de las bombas de expulsión ${ }^{24}$.

Al analizar la resistencia a carbapenémicos, se pudo comprobar que la CIM de IPM y MEM fue mayor en los aislados productores de carbapenemasas que aquellos no productores, con valores de $\mathrm{CIM}_{50 / 90}$ de $256 \mu \mathrm{g} / \mathrm{mL}$, para aislados KPC (+), lo que está en concordancia con un estudio realizado en Estados Unidos de América, que informa la misma situación para enterobacterias ${ }^{16}$, aunque en ese trabajo los valores de CIM de estos carbapenémicos eran más bajos. Estos hallazgos dan cuenta que la producción de carbapenemasas otorga grados más elevados de resistencia. Desde el punto de vista clínico, una alternativa para el tratamiento de infecciones producidas por $P$. aeruginosa productoras de KPC es la combinación ceftazidima/avibactam; sin embargo, el gran problema para el laboratorio, y por ende para el médico clínico, son aquellos aislados que son productores de metalo- $\beta$-lactamasas, como la enzima tipo VIM, que son resistentes a la combinación ceftazidima/avibactam ${ }^{25}$. De ahí la importancia de poder determinar qué tipo de carbapenemasa es la que está sintetizando el aislado resistente al carbapenémico y no sólo determinar la producción de una carbapenemasas por la prueba fenotípica CarbaNP. En general esta prueba fenotípica tiene un elevado rendimiento con una sensibilidad y especificidad de $98 \%$ cuando se estudió en $P$. aeruginosa ${ }^{26}$, como también se observó en este trabajo, y tiene un rendimiento más bajo en $A$. baumannii, por la producción de carbapenemasas del grupo OXA, que ha ido mejorando con diferentes variantes del método. En general, el mayor problema es la detección de OXA-48 que epidemiológicamente es importante en Enterobacterales en España ${ }^{27}$, por ejemplo, pero poco informada hasta ahora en Chile ${ }^{28}$.

Por otra parte, la diseminación del mecanismo de resistencia a carbapenémicos, mediado por carbapenemasas en $P$. aeruginosa, es lo que más preocupa, debido a su creciente prevalencia y su naturaleza fácilmente transmisible. 
Esto último debido a que los genes que las codifican se transportan en elementos genéticos móviles, que también portan genes de resistencia a otros agentes antibacterianos, otorgándoles el carácter de cepas multi-resistentes a los antimicrobianos ${ }^{29}$. Todas las cepas fueron no resistentes a colistina, lo que está en concordancia con muchos estudios que informan una elevada susceptibilidad a esta polimixina entre las cepas de $P$. aeruginosa resistentes a carbapenémicos, con valores de susceptibilidad por sobre $90 \%$ de las cepas ${ }^{6}$.

El estudio de tipificación molecular de los aislados productores de carbapenemasas da cuenta que existe policlonalidad entre ellas, distinguiendo 7 clústeres con sólo dos cepas clonales en el grupo productor de KPC. No existe relación genética entre aislados productores de KPC y VIM y los clústeres están formados por un solo tipo de aislados (productor de KPC o productor de VIM). Por otra parte, sólo en dos clústeres se agruparon aislados de dos ciudades (Temuco y Santiago), lo que podría indicar que las cepas relacionadas genéticamente se distribuyen mayoritariamente sólo en el hospital de origen. Esta policlonalidad también había sido observada en trabajos previos ${ }^{5,6}$ que describen la producción de VIM en cepas de $P$. aeruginosa provenientes de un hospital de Santiago de Chile. La policlonalidad de los aislados encontrados en este estudio podría también ser evidencia que la diseminación de las carbapenemasas entre los aislados de $P$. aeruginosa recuperados en Chile está mediada principalmente por transferencia horizontal de genes (THG), más que por diseminación de un clon productor de estas enzimas.

Aunque estos resultados nos permiten evidenciar el aumento de aislados de $P$. aeruginosa productores de carbapenemasas y dar cuenta que probablemente la emergencia de aislados $\mathrm{KPC}(+)$ sea producto de la adquisición de elementos genéticos móviles por transferencia horizontal genética (THG), desde Klebsiella pneumoniae, que es la Enterobacteriaceae que con mayor frecuencia se ha descrito produciendo este tipo de enzimas en Chile, es necesario continuar estudios que diluciden cómo está ocurriendo la dinámica de la diseminación de carbapenemasas en este importante patógeno causante de IAAS.

\section{Referencias bibliográficas}

1.- Paz-Zarza V M, Mangwani-Mordani S, Martínez-Maldonado A, Álvarez-Hernández D, Solano-Gálvez S G, Vázquez-López R. Pseudomonas aeruginosa: pathogenicity and antimicrobial resistance in urinary tract infection. Rev Chilena Infectol. 2019; 36: 180- 9. http:// dx.doi.org/10.4067/S0716-10182019000200180.

2.- $\quad$ Pang Z, Raudonis R, Glick B R, Lin T J, Cheng Z Antibiotic resistance in Pseudomonas aeruginosa mechanisms and alternative therapeutic strategies Biotechnol Adv. 2019; 37: 177-92. doi: 10.1016/j. biotechadv.2018.11.013.

3.- WHO 2017. World Health Organization-Global priority list of antibiotic-resistant bacteria to guide research, discovery, and development of new antibiotics. https://www.who.int/medicines/ publications/WHO-PPL-Short_Summary_25FebET_NM_WHO.pdf?ua=1.

4.- Otaíza, F, Orsini M, Pohlenz M, Tarride T. Informe de Vigilancia de Infecciones Asociadas a la Atención en Salud. Ministerio de Salud (MINSAL) de Chile 2017; 80. https://www.minsal.cl/wpcontent/uploads/2017/09/informe-vigilancia-2015. pdf.

5.- Pérez I A, García C P, Poggi M H, Braun J S, Castillo V C, Román J C, et al. Presence of metallo beta-lactamases in imipenem-resistant Pseudomonas aeruginosa. Rev Med Chile 2008; 136: 423-32. http://dx.doi.org/10.4067/S003498872008000400002.

6.- Labarca J A, Salles M J, Seas C, GuzmánBlanco M. Carbapenem resistance in
Pseudomonas aeruginosa and Acinetobacter baumannii in the nosocomial setting in Latin America. Crit Rev Microbiol. 2013; 42: 276-92 doi: 10.3109/1040841X.2014.940494.

7.- Wozniak A, Paillavil B, Legarraga P, Zumarán C, Prado S, García P. Evaluation of a rapid immunochromatographic test for detection of $\mathrm{KPC}$ in clinical isolates of Enterobacteriaceae and Pseudomonas species. Diag Microbiol Infect Dis. 2019; 95: 131-3. doi: 10.1128/JCM.01927-19.

8.- CLSI. Methods for dilution antimicrobia susceptibility tests for bacteria that grow aerobically; approved standard-tenth edition. CLSI document M07-A10. Wayne, PA: Clinical and Laboratory Standards Institute 2015.

9.- CLSI. Performance standards for antimicrobial susceptibility testing. 29th ed. CLSI supplement M100. Wayne, PA: Clinical and Laboratory Standards Institute 2019.

10.- Servicio Antimicrobianos, INEI ANLIS “Dr. Carlos G. Malbrán”. Método de screening "COLISTIN AGAR-SPOT" version 2. 2017. http://antimicrobianos.com.ar/ATB/wp-content/ uploads/2017/09/Protocolo-Agar-spot- COL-2017version2-Agosto2017.pdf.

11.- Dortet L, Bréchard L, Poirel L, Nordmann P. Rapid detection of carbapenemase- producing Enterobacteriaceae from blood cultures. Clin Microbiol Infect. 2014; 20: 340-344 doi: 10.1111/1469-0691.12318.

12.- Poirel L, Walsh T R, Cuvillier V, Nordmann P. Multiplex PCR for detection of acquired carbapenemase genes. Diagn Microbiol Infect Dis. 2011; 70: 119-23. doi: 10.1016/j. diagmicrobio.2010.12.002.

13.- Jordan K, Dalmasso, M (eds.), Pulse field gel electrophoresis: methods and protocols, methods in molecular biology, vol. 1301. doi 10.1007/9781-4939-2599-5_14, Springer Science+Business Media New York 2015.

14.- Gomila M. Emergence of carbapenemases in Pseudomonas aeruginosa: a worldwide problem. Expert Rev. Anti Infect. Ther. 2014; 12: 9-11 doi: 10.1586/14787210.2014.866037.

15.- Jing X, Zhou H, Min X, Zhang X, Yang Q, Du $\mathrm{S}$, et al. The simplified carbapenem inactivation method (sCIM) for simple and accurate detection of carbapenemase- producing gram-negative bacilli. Front. Microbiol. 2018; 9: 2391. doi: 10.3389/fmicb.2018.02391.

16.- Papagiannitsis C C, Medvecky M, Chudejova K, Skalova A, Rotova V, Spanelova P, et al. Molecular characterization of carbapenemase-producing Pseudomonas aeruginosa of Czech origin and evidence for clonal spread of extensively resistant sequence type 357 expressing IMP-7 metallo$\beta$-lactamase. Antimicrob Agents Chemother. 2017; 61: e01811-17. https://doi.org/10 .1128/ AAC.01811-17.

17.- Tamma P D, Huang Y, Opene B N A, Simner P J. Determining the optimal carbapenem MIC that distinguishes carbapenemase-producing and non-carbapenemase-producing carbapenemresistant Enterobacteriaceae. Antimicrob Agents Chemother. 2016; 60: 6425-9. doi:10.1128/ AAC.00838-16.

18.- Mendes R, García P, Guzmán M, Toleman M, Walsh T, Jones R. First isolation of blaVIM-2 
in Latin America: Report from the SENTRY Antimicrobial Surveillance Program. Antimicrob Agents Chemother. 2004; 48: 1433-4. doi: 10.1128/aac.48.4.1433-1434.2004.

19.- Gastelo-Acosta R M, Díaz-Sipión R S, Maguiña Vargas C. Carbapenemasas en bacterias Gram negativas no fermentadoras aisladas en servicios críticos del Hospital Regional Lambayeque, diciembre 2014 - julio 2015. Acta Médica Peruana. 2016; 33: 183-8. http:/www.scielo.org.pe/pdf/ amp/v33n3/a03v33n3.pdf.

20.- Cornaglia G, Giamarellou H, Rossolini G M. Metallo- $\beta$-lactamases: a last frontier for $\beta$ lactams? Lancet Infect Dis. 2011; 11: 381-93. doi: 10.1016/S1473-3099(11)70056-1.

21.- Kazmierczak K M, Biedenbach D J, Hackel M, Rabine S, de Jonge B L, Bouchillon S K, et al. Global dissemination of blaKPC into bacterial species beyond Klebsiella pneumoniae and in vitro susceptibility to ceftazidime-avibactam and aztreonam- avibactam. Antimicrob Agents Chemother. 2016; 60: 4490-500. doi: 10.1128/ AAC.00107-16.

22.- Ide D, Aguayo K, De la Cerda G, Medel M, Riquelme M I, Román JC, et. al. Vigilancia de bacilos gramnegativos productores de carbapenemasas en pacientes trasladados desde otras instituciones: Perspectiva de 3 años. Rev Chilena Infectol. 2017; 34 (Supl 1): s51. Abstract P-50. http://www.sochinf.cl/portal/templates/ sochinf2008/documentos/2017/Rev_Congreso_ Infect.pdf.

23.- Pacheco T, Bustos-Cruz R H, Abril D, Arias S, Uribe L, Rincón J, et. al. Pseudomonas aeruginosa coharboring blaKPC-2 and blaVIM-2 carbapenemase genes. Antibiotics (Basel). 2019; 20: 8 (3). pii: E98. doi: 10.3390/ antibiotics 8030098

24.- Kresken M, Körber-Irrgang B, Korte-Berwanger M, Pfennigwerth N, Gatermann S G, Seifert H. German Carbapenem Resistance Study Group. Dissemination of carbapenem-resistant Pseudomonas aeruginosa isolates and their susceptibilities to ceftolozane-tazobactam in Germany. Int J Antimicrob Agents. 2020; 55 (6): 105959. doi: 10.1016/j.ijantimicag.2020.105959.

25.- Doi Y. Treatment options for carbapenem-resistant gram-negative bacterial infections. Clin Infect Dis. 2019; 69 (Suppl 7): S565-S575. doi: 10.1093/cid/ ciz830.
26.- Tamma P D, Simner P J. Phenotypic detection of carbapenemase-producing organisms from clinical isolates. J Clin Microbiol. 2018; 56 (11): e0114018. doi: 10.1128/JCM.01140-18.

27.- Mora-Guzmán I, Rubio-Pérez I, Domingo-García D, Martín-Pérez E. Infecciones asociadas a enterobacterias productoras de carbapenemasas OXA-48 en pacientes quirúrgicos: consumo de antibióticos y evolución de sensibilidades. Rev Esp Quimioter. 2020; 33 (6): 448-52. doi: 10.37201/ req/081.2020.

28.- Carrasco-Anabalón S, Conceição Neto C O, D'Alincourt Carvalho-Assef A P, Lima CA, Cifuentes M, Silva F, et al. Introduction of NDM-1 and OXA-370 from Brazil into Chile in strains of Klebsiella pneumoniae isolated from a single patient. Int J Infect Dis. 2019; 81: 28-30. doi: 10.1016/j.ijid.2019.01.051.

29.- Schäfer E, Malecki M, Tellez-Castillo C J, Pfennigwerth N, Marlinghaus L, Higgins P G, et al. Molecular surveillance of carbapenemaseproducing Pseudomonas aeruginosa at three medical centres in Cologne, Germany. Antimicrob Res Infect Control 2019; 8: 208 https://doi. org/10.1186/s13756-019-0665-5. 